\title{
Epidemiology of Bronchopulmonary Dysplasia Among Preterm Infants in Saudi Arabia
}

\author{
Saleh Algarni \\ College of applied medical science. King Saud bin Abdulaziz \\ University for Health Sciences Saudi Arab
}

\section{ABSTRACT}

Despite the overall improvement in preterm survival over the last years, more of these survivors have diagnosed with Bronchopulmonary dysplasia (BPD). BPD remains one of the most common conditions affecting preterm infants and linked with long-term impact on infant, family, healthcare system, and economy. The BPD incidence rate in Saudi Arabia was underreported in the literature conducted at a local level. The rate fluctuated between $17.7 \%$ and $36.5 \%$. However, the national prevalence of BPD is not established yet. Therefore, an overall incidence rate of BPD in Saudi Arabia need to be established and therefore the burden of BPD can be studied and possibly minimised.

KEY WORDS: BPD, PRETERM, RESPIRATORY, BURDEN, SAUDI.

\section{INTRODUCTION}

Preterm infants are at risk of developing respiratory complications specific to factors associated with prematurity and medical management. The most common respiratory complication is Bronchopulmonary Dysplasia (BPD). This condition is mainly managed in the neonatal intensive care unit (NICU) with infants in hospital for many months. BPD is a serious complication unique to preterm infants. In 1960, Northway described BPD as the radiological and clinical abnormalities associated with the presence of RDS that occurred in moderate to late preterm infants. In 1985, O'Brodovich and Mellins revised this with BPD more clearly defined as inflammation, fibrosis, and hypertrophy of the smooth muscles caused by oxidant injury and mechanical ventilation (MV). Common risk factors leading to BPD are prematurity, oxygen toxicity,

Biosc Biotech Res Comm P-ISSN: 0974-6455 E-ISSN: 2321-4007

\section{crossef}

Identifiers and Pagination

Year: 2021 Vol: 14 No (5) Special Issue

Pages: 80-83

This is an open access article under Creative

Commons License Attribn 4.0 Intl (CC-BY).

DOI: $h t t p: / / d x . d o i . o r g / 10.21786 / b b r c / 14.5 / 16$ and MV. The length of hospital stay for preterm infants diagnosed with BPD is longer than those without BPD. This review aims to establish the current incidence rate of BPD at Saudi Arabia. By establishing the rate, the approach to overcome the burden of BPD can be tackled and planned.

Definition of BPD: Historically, there was discrepancy in identifying criteria for defining BPD. The earliest definition was based on radiological changes and oxygen requirement only. However, a severity-based definition of BPD considers total duration of supplemental oxygen requirement, need for positive pressure ventilation, and gestational age. For preterm infants less than 32 weeks of gestational age: "Mild BPD is defined as a need for supplemental oxygen for $\geq 28$ days but not at 36 weeks' PMA or discharge, moderate BPD as oxygen for $\geq 28$ days plus treatment with $<30 \%$ oxygen at 36 weeks' PMA, and severe BPD as oxygen for $\geq 28$ days plus $\geq 30 \%$ oxygen and/or positive pressure at 36 weeks' PMA".

However, this widely used definition may no longer be valid because it does not account for changes in respiratory support management such as the widespread use of high flow (HF) and incidence of long-term respiratory morbidities for preterm infants. Therefore, a recent revision for this definition was conducted to

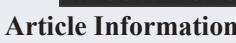

Received: $15^{\text {th }}$ Jan 2021

ccepted after revision: $27^{\text {th }}$ Mar 2021

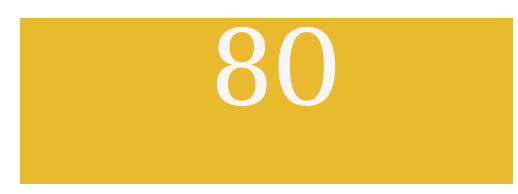


develop an evidence-based definition. This proposed definition defines BPD based on the respiratory support mode administered at 36 weeks PMA regardless of previous oxygen therapy. BPD severity was ranked as follows: no support = no BPD, nasal cannula $\leq 2 \mathrm{~L} / \mathrm{min}$ = grade $1 \mathrm{BPD}$, nasal cannula $>2 \mathrm{~L} / \mathrm{min}$ or non-invasive ventilation = grade $2 \mathrm{BPD}$, and invasive ventilation = grade $3 \mathrm{BPD}$.

Pathophysiology of BPD and Common Risk Factors: The lungs of preterm infants born at early gestations are immature and still undergoing development. Thus, immature lungs do not support efficient gas exchange and any injury of the lung at this stage will affect lung growth and potentially lead to long term pulmonary complications. The pathogenesis of BPD is multifactorial and risk factors are categorised into either pre or postnatal causes. The most significant factor associated with BPD incidence is prematurity. In addition, other factors can contribute to the development of BPD including positive pressure ventilation, oxygen toxicity, and pre/post-neonatal infection. All of these factors can separately or jointly provoke an inflammatory response within the lung.

In normal lung development, alveolarisation starts during late fetal development and continues into the early stage of childhood. In BPD, the alveolarisation is impaired and lung inflammation will be induced by either infectious agents such as viral infection or non-infectious factors such as high oxygen administration and positive pressure ventilation. During the inflammatory response, the release of cytokines and disturbance of growth factor signalling will result in an increase in lung tissue damage and cell apoptosis, which impact all different cell types, and cause inactivation of surfactant. Surfactant inactivation is a key cause of the RDS development in preterm infants.

Prematurity and BPD: In premature infants, BPD is considered the most common cause of respiratory morbidity. BPD incidence is inversely related to gestational age and birth weight. Therefore, the risk of BPD is greatly increased among extremely preterm and very low birthweight infants.

Oxygen Toxicity and BPD: The toxic effects that excess oxygen can have on the newborn, particularly if preterm, are well described and known to contribute to significant morbidities such as BPD and retinopathy of prematurity (ROP). Awareness of the toxicity of oxygen has been increasing in neonatal medicine over the last few decades. Oxidative stress in premature infants occurs due to the free radicals generated by oxygen, which are not handled well by their undeveloped antioxidant systems.

Therefore, the imbalance between oxidant and antioxidant factors leads to oxidative stress. Studies conducted by exposing rats and mice to 50\% or more oxygen result in the inhibition of pulmonary growth and DNA synthesis. The administration of oxygen can compromise the development of the lung, as high levels of oxygen administration can result in free radical production, injuring the lung and resulting in long-term morphological changes including cessation of cell growth and development.

Mechanical Ventilation and BPD: In NICU settings, MV is commonly used as a lifesaving intervention, especially for respiratory failure associated with prematurity. However, the use of positive pressure ventilation and high tidal volumes can cause pulmonary baro/volutrauma, which results in a reduction in lung compliance and the development of lung injury. The need of MV in managing preterm infants has been found to be linked with the development of BPD. In addition, the length of MV correlates with the development of BPD. Therefore, by improving respiratory management practices and avoiding MV, BPD could be reduced. MV affects the dynamic characteristics of the preterm lung, as the decreased compliance caused by surfactant deficiency contributes to non-uniform lung expansion with areas over distended and others collapsed. Therefore, if the premature lung is over inflated via positive pressure ventilation and excess volume, this can lead to cellular injury and inflammation.

The Burden of BPD: Despite improvements in the survival of infants born $<32$ weeks' gestation over the last two decades, more of these survivors have a diagnosis of BPD. BPD remains one of the most common conditions affecting preterm infants and links with long-term impact on child health, the family, healthcare systems, and the economy.

Health Long-Term Outcomes of BPD: As the survival rate of preterm infants improves, more survivors will develop BPD. BPD infants suffer from many neurodevelopmental problems; survivors with BPD assessed at preschool and school age were found to perform below average on cognitive, psychomotor, and linguistic evaluation. The literature describes the long-term consequences of BPD as a crucial predictor of long-term respiratory outcomes in child and adulthood. Preterm survivors with BPD were found to have significantly impaired lung function and an increased risk of asthma compared to those without BPD. In addition, Pulmonary diseases developed in adulthood such as asthma and chronic obstructive pulmonary disease have been associated with preterm birth.

BPD Burden on Family: Parents play an important role in caring for BPD neonate. The short and long-term health and cognition outcomes for BPD neonate impose a significant burden on family to control and minimise the complications associated with BPD. Overall, parents having preterm infants reported to have social isolation, negative consequence in workplace, and financial burden.

BPD Burden on Healthcare System and Economy: The economic burden of BPD is understudied. There were only two studies described it at national level in UK and 
Canada. The EPICure UK study reported that an extremely preterm infant in the 11th year of life has significantly higher annual healthcare, social care and educational cost differences of approximately £2,500 per annum (approximately 12,500 SAR) compared to term-matched child, the cost was expressed for the financial year 20062007. A recent financial model from Canada has been developed to estimate the lifetime economic burden of an extremely preterm infant diagnosed with BPD. The model reported that an infant with BPD during their lifetime incurs over 700,000 Canadian dollars (approximately 2 million SAR) from healthcare costs.

BPD in Saudi Arabia: The prevalence and incidence of BPD are understudied in Saudi Arabia. Up to the best of my knowledge there were only limited studies that report BPD incidence at local level using retrospective approaches. In 2011, Alhazzani et al reported the outcome of very low birthe weight infants admitted to neonatal unit at a tertiary hospital in Riyadh during the period from January 2006 to December 2008. They define BPD as need of oxygen at 36 weeks of PMA, with a previous need of oxygen of at least 28 days, the BPD rate found to be $17.7 \%$ (i.e. 33 out of 186 infants included in this study). In 2018, Abolfotouh et al reported the early and late outcomes of extremely low birth weight in one tertiary hospital in Riyadh during the period from January 2005 to December 2007. The BPD defined as oxygen dependency at 36 weeks of PMA only. The BPD rate found to be $36.5 \%$ (i.e. 42 out of 117 infants included).

A retrospective cohort study in a single tertiary neonatal centre in Riyadh was conducted to assess premature infants' outcome during the period from January 2015 to December 2019. The BPD defined as oxygen dependency at 36 weeks of PMA or at oxygen if discharge before at 34 and 35 weeks of PMA, the rate found to be around $20 \%$ for Saudi infants. The Majority of published studies report the BPD incidence in tertiary hospitals placed in the capital city. The limited number of studies describing BPD incidence is probably because of the absence of a national neonatal database. In addition to the lack of multicentre collaboration in researches between neonatal units. The development of the information technology sector in Saudi Arabia is hugely growing over the last years ,especially after the implementation of the Kingdom 2030 vision.

Technology is a keystone of this valuable vision. The E-government initiatives of Saudi Arabia are progressing and successfully attained their goals in many governmental ministries, including the ministry of Health. The need for the national neonatal database is critically important to monitor and aid in both regional and national quality improvement of newborn infants in Saudi Arabia. Example of the national data uses include describing the prevalence of preterm birth, evaluate the impact of quality project at regional level, and support developing researches in Saudi Arabia and participation in multicentre international studies.

\section{CONCLUSION}

The incidence of BPD in Saudi Arabia is not adequately and accurately reported. The need for a Saudi national neonatal database is vital to form a unique, reliable resource to report neonatal outcomes, including BPD. This resource will support local, regional, national, and international work ranging from quality project to observational and interventional studies. This database can also monitor the overall outcome of neonates in Saudi Arabia and support higher governmental decision based on these outcomes.

\section{REFERENCES}

Abolfotouh, M.A., et al., Prospective study of early and late outcomes of extremely low birthweight in Central Saudi Arabia. BMC Pediatr, 2018. 18(1): p. 280.

Al Hazzani, F., et al., Short-term outcome of very lowbirth-weight infants in a tertiary care hospital in Saudi Arabia. Ann Saudi Med, 2011. 31(6): p. 581-5.

Al-Mouqdad, M., et al., Antenatal care of mothers and morbidity and mortality disparities among preterm Saudi and non-Saudi infants less than or equal to 32 weeks' gestation. Ann Saudi Med, 2020. 40(4): p. 290297.

Avery, M.E., et al., Is chronic lung disease in low birth weight infants preventable? A survey of eight centers. Pediatrics, 1987. 79(1): p. 26-30.

Bancalari, E. and T. Gerhardt, Bronchopulmonary dysplasia. Pediatr Clin North Am, 1986. 33(1): p. 1-23.

Chakraborty, M., E.P. McGreal, and S. Kotecha, Acute lung injury in preterm newborn infants: mechanisms and management. Paediatr Respir Rev, 2010. 11(3): p. 162-70; quiz 170.

Costeloe, K.L., et al., Short term outcomes after extreme preterm birth in England: comparison of two birth cohorts in 1995 and 2006 (the EPICure studies). Bmj, 2012. 345: p. e7976.

Cotten, C.M., et al., Prolonged hospital stay for extremely premature infants: risk factors, center differences, and the impact of mortality on selecting a best-performing center. J Perinatol, 2005. 25(10): p. 650-5.

Davidson, L.M. and S.K. Berkelhamer, Bronchopulmonary Dysplasia: Chronic Lung Disease of Infancy and LongTerm Pulmonary Outcomes. Journal of clinical medicine, 2017. 6(1): p. 4.

Doyle, L.W. and P.J. Anderson, Long-term outcomes of bronchopulmonary dysplasia. Semin Fetal Neonatal Med, 2009. 14(6): p. 391-5.

Ehrenkranz, R.A., et al., Validation of the National Institutes of Health consensus definition of bronchopulmonary dysplasia. Pediatrics, 2005. 116(6): p. 1353-60.

Gien, J. and J.P. Kinsella, Pathogenesis and treatment of bronchopulmonary dysplasia. Curr Opin Pediatr, 2011. 23(3): p. 305-13.

Gough, A., et al., Impaired lung function and health 
status in adult survivors of bronchopulmonary dysplasia. Eur Respir J, 2014. 43(3): p. 808-16.

Halliday, H.L., The fascinating story of surfactant. J Paediatr Child Health, 2017. 53(4): p. 327-332.

Han, R.N., et al., Changes in structure, mechanics, and insulin-like growth factor-related gene expression in the lungs of newborn rats exposed to air or 60\% oxygen. Pediatr Res, 1996. 39(6): p. 921-9.

Hilgendorff, A. and M.A. O'Reilly, Bronchopulmonary dysplasia early changes leading to long-term consequences. Front Med (Lausanne), 2015. 2: p. 2.

Islam, J.Y., et al., Understanding the Short- and Long-Term Respiratory Outcomes of Prematurity and Bronchopulmonary Dysplasia. Am J Respir Crit Care Med, 2015. 192(2): p. 134-56.

Jensen, E.A., et al., Effects of Multiple Ventilation Courses and Duration of Mechanical Ventilation on Respiratory Outcomes in Extremely Low-Birth-Weight Infants. JAMA pediatrics, 2015. 169(11): p. 1011-1017. Jensen, E.A., et al., The Diagnosis of Bronchopulmonary Dysplasia in Very Preterm Infants. An Evidence-based Approach. American journal of respiratory and critical care medicine, 2019. 200(6): p. 751-759.

Jobe, A.H., et al., Injury and inflammation from resuscitation of the preterm infant. Neonatology, 2008. 94(3): p. 190-6.

Kusters, C.D., et al., [The impact of a premature birth on the family; consequences are experienced even after 19 years]. Ned Tijdschr Geneeskd, 2013. 157(25): p. A5449.

Moss, T.J., Respiratory consequences of preterm birth. Clin Exp Pharmacol Physiol, 2006. 33(3): p. 280-4.

Muscedere, J.G., et al., Tidal ventilation at low airway pressures can augment lung injury. Am J Respir Crit Care Med, 1994. 149(5): p. 1327-34.

Northway, W.H., Jr., R.C. Rosan, and D.Y. Porter, Pulmonary disease following respirator therapy of hyaline-membrane disease. Bronchopulmonary dysplasia. N Engl J Med, 1967. 276(7): p. 357-68.

0’Brodovich, H.M. and R.B. Mellins, Bronchopulmonary dysplasia. Unresolved neonatal acute lung injury. Am Rev Respir Dis, 1985. 132(3): p. 694-709.

Perez Tarazona, S., et al., Bronchopulmonary dysplasia as a risk factor for asthma in school children and adolescents: A systematic review. Allergol Immunopathol (Madr), 2018. 46(1): p. 87-98.

Petrou, S., et al., Costs and health utilities associated with extremely preterm birth: evidence from the EPICure study. Value Health, 2009. 12(8): p. 1124-34.
Poindexter, B.B., et al., Comparisons and Limitations of Current Definitions of Bronchopulmonary Dysplasia for the Prematurity and Respiratory Outcomes Program. Ann Am Thorac Soc, 2015. 12(12): p. 1822-30.

Russell, R.B., et al., Cost of hospitalization for preterm and low birth weight infants in the United States. Pediatrics, 2007. 120(1): p. e1-9.

Santhakumaran, S., et al., Survival of very preterm infants admitted to neonatal care in England 20082014: time trends and regional variation. Arch Dis Child Fetal Neonatal Ed, 2018. 103(3): p. F208-f215.

Saugstad, O.D., Chronic lung disease: the role of oxidative stress. Biol Neonate, 1998. 1: p. 21-8.

Shahzad, T., et al., Pathogenesis of bronchopulmonary dysplasia: when inflammation meets organ development. Molecular and cellular pediatrics, 2016. 3(1): p. 2323.

Short, E.J., et al., Developmental sequelae in preterm infants having a diagnosis of bronchopulmonary dysplasia: analysis using a severity-based classification system. Arch Pediatr Adolesc Med, 2007. 161(11): p. 1082-7.

Siffel, C., et al., Global incidence of bronchopulmonary dysplasia among extremely preterm infants: a systematic literature review. J Matern Fetal Neonatal Med, 2019: p. 1-11.

Sola, A., et al., Safe oxygen saturation targeting and monitoring in preterm infants: can we avoid hypoxia and hyperoxia? Acta Paediatrica, 2014. 103(10): p. 1009-1018.

Sola, A., M.R. Rogido, and R. Deulofeut, Oxygen as a neonatal health hazard: call for detente in clinical practice. Acta Paediatr, 2007. 96(6): p. 801-12.

Stoll, B.J., et al., Neonatal outcomes of extremely preterm infants from the NICHD Neonatal Research Network. Pediatrics, 2010. 126(3): p. 443-56.

van Katwyk, S., et al., Lifetime patient outcomes and healthcare utilization for Bronchopulmonary dysplasia (BPD) and extreme preterm infants: a microsimulation study. BMC pediatrics, 2020. 20(1): p. 136-136.

Van Marter, L.J., et al., Do clinical markers of barotrauma and oxygen toxicity explain interhospital variation in rates of chronic lung disease? The Neonatology Committee for the Developmental Network. Pediatrics, 2000. 105(6): p. 1194-201.

Wilborn, A.M., L.B. Evers, and A.T. Canada, Oxygen toxicity to the developing lung of the mouse: role of reactive oxygen species. Pediatr Res, 1996. 40(2): p. 225-32. 